\title{
The metabolic syndrome entanglement: Cutting the Gordian knot
}

\author{
Enrique Z. Fisman ${ }^{1,2}$, Alexander Tenenbaum ${ }^{1,2,3}$ \\ ${ }^{1}$ Sackler Faculty of Medicine, Tel-Aviv University, Tel-Aviv, Israel \\ ${ }^{2}$ Cardiovascular Diabetology Research Foundation, Holon, Israel \\ ${ }^{3}$ Cardiac Rehabilitation Institute, Sheba Medical Center, Tel-Hashomer, Israel
}

\begin{abstract}
Questions have been raised on the clinical value of the metabolic syndrome (MS). The negative opinion regarding $M S$ is anchored basically on a separate analysis of 4 conditions: obesity, dyslipidemia, hypertension and glucose intolerance. The common denominator of these 4 sets of arguments is that they represent an utterly simplistic view of MS as a solely predictive tool of morbidity or mortality. We believe that it is inequitable to compare it with statistically constructed predictive tools, including stronger prognostic variables even unrelated to one another from the biological point of view. Several recent large meta-analyses - one of them including nearly one million patients - systematically showed that people with MS are at increased risk of cardiovascular (CV) events. MS was associated with a 2-fold increase in CV outcomes and a 1.5-fold increase in all-cause mortality rates. A very important finding was that $C V$ risk still remained high in patients with MS but without diabetes. The presence of MS possesses a definitely predictive value, but above all it is a widely accepted concept regarding a biological condition based on complex and interrelated pathophysiological mechanisms emanating from excess central adiposity and insulin resistance. The risk factors are multiplicative, meaning that the risk of a $C V$ disease from risk factors rises geometrically, not linearly, as the number of risk factors increases. Therefore, currently available evidences strongly support the concept of the MS as a critical clustering of $C V$ risk factors and diabetes, representing a true and solid evolving clinical entity. (Cardiol J 2014; 21, 1: 1-5)
\end{abstract}

Key words: cardiovascular disease, dyslipidemia, hypertension, insulin resistance, metabolic syndrome, obesity, type 2 diabetes mellitus

\section{Introduction}

During the last few years, an enormous amount of articles have been published regarding a relatively new diagnostic category: the metabolic syndrome (MS). These articles have proliferated in medical literature in the fields of internal medicine, cardiology, endocrinology, metabolism, hypertension and related disciplines. Initially, in 1988 Reaven [1] proposed a conceptual framework which linked several apparently unrelated biological events into a single pathophysiological assemble. His hypothesis argued that insulin resistance triggered a common mechanism underlying the associated abnormalities of blood pressure, high density lipoprotein cholesterol (HDL-C), triglycerides (TG) and glucose tolerance. This concept subsequently evolved to encompass a number of multiple definitions [2] established by the World Health Organization (WHO) [3], the National

Address for correspondence: Prof. Enrique Z. Fisman, MD, Cardiovascular Diabetology Research Foundation, 58484 Holon, Israel, tel: 97252 7843579, e-mail zfisman@post.tau.ac.il

Received: 02.04.2013 Accepted: 02.04.2013 
Cholesterol Education Program Adult Treatment Panel III (ATP III) [4], the European Group for the Study of Insulin Resistance [5] and the International Diabetes Federation (IDF) [6]. Central obesity has been added to the above mentioned risk factors, which were ranked in different pathogenic priorities and with dissimilar cut-off points for the individual risk factors [7]. More recently, the IDF and the modified ATP III definitions have become more concordant, with the remaining difference pertaining to waist measurement [8].

However, questions have been raised concerning the clinical value of the MS [9]. Perhaps the most direct assault on the theory has been issued in a joint report [10] from the American Diabetes Association (ADA) and the European Association for the Study of Diabetes (EASD), which questions the very existence of MS. In sharp contrast, a joint position paper from the American Heart Association and the National Heart, Lung, and Blood Institute, firmly defends the concept [11].

In the present review we deal with the pros and cons of this issue. We will firstly briefly describe the main arguments against the survival of $\mathrm{MS}$ as an independent clinical entity. Then, we are going to offer the current evidence to the contrary, in an attempt to cut the Gordian knot around the MS entanglement.

\section{Cons}

The negative opinion regarding the syndrome is anchored on the analysis of 4 conditions: obesity, dyslipidemia, hypertension and glucose intolerance.

First, regarding obesity it may be argued that excess adiposity is not a consequence of insulin resistance, but a change that increases the likelihood that an individual will be insulin resistant. In addition, not all obese individuals are insulin resistant [12] and, as a corollary, the risk factors for cardiovascular disease (CVD) should be confined to the subset of obese individuals who are also insulin resistant [13]. Obesity, per se, in the absence of the metabolic characteristics of insulin resistance, should not increase the incidence of either CVD or type 2 diabetes mellitus (T2DM) [14].

Second, there is a highly significant relationship between insulin resistance, compensatory hyperinsulinemia, and hypertriglyceridemia [15], which is not limited to an increase in plasma TG concentrations. Thus, although various definitions of MS have selected the combination of a high plasma TG and a low HDL-C concentration as diagnostic criteria, these changes are also associated with a decrease in low-density lipoprotein (LDL) and the postprandial accumulation of TG-rich remnant lipoproteins [16]. Both high TG and low HDL-C have been shown to increase risk of CVD [17] and may develop for reasons other than insulin resistance/hyperinsulinemia.

Third, essential hypertension is intimately linked to insulin resistance/hyperinsulinemia. Firstly, as a group, patients with essential hypertension are insulin resistant and hyperinsulinemic [18]. Secondly, normotensive first-degree relatives of patients with essential hypertension are relatively insulin resistant and hyperinsulinemic as compared to a matched control group without a family history of hypertension [19]. Thirdly, hyperinsulinemia, as a surrogate estimate of insulin resistance, has been shown in population-based studies to predict the eventual development of essential hypertension [20]. These data provide substantial support for the theory that insulin resistance/hyperinsulinemia plays a role in the pathogenesis of essential hypertension [9]. On the other hand, probably no more than $50 \%$ of patients with essential hypertension are insulin resistant [21]. However, it is only this subset of patients that display the components of the various definitions of MS that render them at greatest risk for CVD [9].

Fourth, it is well established that prevalence of some degree of abnormal glucose tolerance and/or T2DM is the abnormality most closely related to insulin resistance. More than 70 years ago Himsworth and Kerr presented evidence that "a state of diabetes might result from inefficient action of insulin as well as from a lack of insulin" [22]. Nowadays it is quite clear that resistance to insulin-mediated glucose disposal is present in great majority of individuals with T2DM [23], and that insulin resistance (or hyperinsulinemia as a surrogate estimate of insulin resistance) is a powerful and independent predictor of the development of T2DM [24].

Therefore, taking into consideration the 4 claims, the criteria for the syndrome will encompass individuals with overt disease (like CVD, T2DM, hypertension), as well as with far milder forms of the same conditions. The definition will capture a spectrum of severities, and it is highly likely that a person who satisfies the diagnostic criteria with risk factor levels just over the cut point will have a much lower CVD risk than another individual with the same combination but higher risk factor levels [10]. 


\section{Pros}

The common denominator of the above mentioned 4 sets of arguments is that they represent an utterly simplistic view of MS as a solely predictive tool of morbidity or mortality [25]. Of course, the presence of MS possesses a definite predictive value, but first of all it is a widely accepted concept regarding a biological condition based on the complex and interrelated pathophysiological mechanisms emanating from excess central adiposity and insulin resistance. Therefore, it is inequitable to compare it with statistically constructed predictive tools, including stronger prognostic variables even unrelated to one another from the biological point of view. For example, in the criteria for MS (in contrast to Framingham score) age and LDL cholesterol (LDL-C) levels are not included, as well as a variety of strong predictors used in other risk stratification scores: previous myocardial infarction, heart failure, smoking, family history, etc. However, MS identifies additional important residual vascular risk mainly associated with insulin resistance and atherogenic dyslipidemia (low HDL-C, high TG, small dense LDL-C). Therefore, MS could be a useful additional tool in estimation of global cardiovascular (CV) risk beyond age, gender, high LDL-C or other standard risk factors.

Additionally, one of the important questions still remaining open is its predictive value: does MS forecast CV events, diabetes onset or disease progression any better than the sum of its components? For the time being, the strongest available evidence points toward a positive answer [25]. Three consecutive large meta-analyses systematically showed that people with MS are at increased risk of CV events [26-28]. The most recent and largest of them [28] included nearly 1 million patients (total $n=951,083$ ). There, the investigators concluded that MS is associated with a 2-fold increase in CV outcomes and a 1.5-fold increase in all-cause mortality rates. That meta-analysis showed that the point estimates for CV risk were consistently higher in women than in men. In addition, a very important finding of this study demonstrated that CV risk was still high in patients with MS but without diabetes. Moreover, it was demonstrated that the syndrome predicts $\mathrm{CV}$ events or/and diabetes independently from other conventional risk factors [29, 30]. Our group has shown that MS is a strong independent predictor of mortality and morbidity in patients with acute coronary syndrome [30]. It should be specifically pointed out that our patients with hyperglycemia and MS had higher mortality rates compared with patients with the same hyperglycemia but without MS (for example 30-day mortality rates respectively $8.3 \%$ vs. $2.5 \%, \mathrm{p}<0.05)$.

Analyzing the progression of carotid atherosclerosis, it should be stressed that subjects with MS have higher levels of intima-media thickness (IMT) and total plaque area at follow up than those without MS. MS predicts progression of IMT in individuals below 50 years of age, but not in older age groups, suggesting thus that MS may be involved in the initial steps of the atherosclerotic process [31].

Another argument in favor of the biological soundness of MS is based on experimental research. For instance, it should be noted that GLUT4 expression, accompanied by whole-body insulin resistance and increased plasma concentration of inflammatory biomarkers was found in an animal model of MS [32], similarly, it has been observed in humans.

Even critics of the MS concept should agree that obesity, dysglycemia, dyslipidemia and hypertension coexist more frequently than predicted by mere chance. These common chronic conditions (and components of MS) have partially overlapping mechanisms of pathogenic actions mediated through common metabolic pathways. Therefore, their total combined effect could be less than the sum of the individual effects [25].

Emerging varied areas of current MS research interests include heterogeneous topics like adiponectin and biomarkers preserving endothelial health [33, 34], angiotensinogen, resistin, and leptin secretion [33], nonalcoholic fatty liver disease and liver steatosis [35], hyperuricemia [36], genetic predisposition [37], the role of adypocytokines and neurohumoral dysregulation [38]; sleep disorders and ethnicity [39], the possible ways for treatment optimization in patients with CVD [40]. Additional related matters are currently investigated, like heart rate (HR) turbulence; it has been shown that this turbulence - a well-established predictor of cardiac autonomic dysfunction - is significantly impaired in MS patients without T2DM in adults [41]. Regarding childhood and adolescence, HR variability is disturbed in children [42], and several equations have been developed in order to early identify and adequately treat MS in young populations, taking into consideration racial, ethnic and gender differences [43]. A multimarker approach to MS could further improve CV risk stratification [44].

The concept that the MS is a consequence of obesity and insulin resistance, provides a useful "life-style changes" approach for prevention and 
treatment: caloric restriction, weight-loss and increased physical activity. The next step could theoretically be pharmacological interventions such as metformin, acarbose, fibrates, weight-loss drugs (like orlistat) and perhaps glucagon-like peptide-1 agonists [45] or eventual new drugs to simultaneously treat several risk factors. A third step is represented by the surgical approach. A recent study authored by Rizzello et al. [46] and performed according to the updated guidelines for meta-analyses of non-randomized studies [47] depicts several promising surgical approaches for MS, including adjustable silicone gastric banding, Roux-en-Y gastric bypass, biliopancreatic diversion and bariatric surgery.

\section{Conclusions}

Currently available evidences strongly support the concept of MS as very far of being factitious. Quite the opposite, it is a critical clustering of CV risk factors and diabetes, representing a true and solid evolving clinical entity. These risk factors are multiplicative, meaning that the risk for CVD from risk factors rises geometrically, not linearly, as the number of risk factors increases. Therefore, the total risk that MS represents is much more than a mere summation of the isolated factors [48, 49]. MS is a multifactorial syndrome, standing firstly on 2 tightly intertwined conditions: obesity and insulin resistance; obesity causes insulin resistance, and on the other hand, insulin resistance modifies adipose tissue responses to insulin and thereby recapitulates the obese state. This situation is exacerbated by the concomitant factors [11, 48]. MS prevention, recognition, treatment, and better understanding of its underlying mechanisms should become an important approach for the reduction of CVD burden in the general population. Future problem-oriented research is needed to improve and unify its diagnostic criteria, its genetic and environmental basis and the optimal medical management.

\section{Acknowledgements}

This work was supported in part by the Cardiovascular Diabetology Research Foundation (RA 58-040-684-1), Holon, Israel.

Conflict of interest: none declared

\section{References}

1. Reaven GM. Banting Lecture 1988. Role of insulin resistance in human disease. Diabetes, 1988; 37:1595-1607.
2. Simmons RK, Alberti KG, Gale EA et al. The metabolic syndrome: Useful concept or clinical tool? Report of a WHO Expert Consultation. Diabetologia, 2010; 53: 600-605.

3. World Health Organization. Definition, diagnosis and classification of diabetes mellitus and its complications. Part 1: Diagnosis and classification of diabetes mellitus. WHO, Geneva, Switzerland, 1999, pp. 1-59.

4. Grundy SM, Brewer HB Jr, Cleeman JI et al. Definition of metabolic syndrome: Report of the National Heart, Lung, and Blood Institute/American Heart Association conference on scientific issues related to definition. Circulation, 2004; 109:433-438.

5. Balkau B, Charles MA. Comment on the provisional report from the WHO consultation. European Group for the Study of Insulin Resistance (EGIR). Diabet Med, 1999; 16: 442-443.

6. Alberti KG, Zimmet P, Shaw J. The metabolic syndrome: A new worldwide definition. Lancet, 2005; 366:1059-1062.

7. Lee IT, Chiu YF, Hwu CM et al. Central obesity is important but not essential component of the metabolicsyndrome for predicting diabetes mellitus in a hypertensive family-based cohort. Results from the Stanford Asia-pacific program for hypertension and insulin resistance (SAPPHIRe) Taiwan follow-up study. Cardiovasc Diabetol, 2012; 11: 43.

8. Alberti KG, Eckel RH, Grundy SM et al. Harmonizing the metabolic syndrome. A Joint Interim Statement of the International Diabetes Federation Task Force on Epidemiology and Prevention; National Heart, Lung, and Blood Institute; American Heart Association; World Heart Federation; International Atherosclerosis Society; and International Association for the Study of Obesity. Circulation, 2009; 120: 1640-1645.

9 Reaven GM, The individual components of the metabolic syndrome: is there a raison d'etre? J Am Coll Nutr, 2007; 26: 191-195.

10. Kahn R, Buse J, Ferrannini E, Stern M. The metabolic syndrome: time for a critical appraisal. Joint statement from the American Diabetes Association and the European Association for the Study of Diabetes. Diabetes Care, 2005; 28: 2289-2304.

11. Grundy SM, Cleeman JI, Daniels SR et al. American Heart Association; National Heart, Lung, and Blood Institute: Diagnosis and management of the metabolic syndrome: An American Heart Association/ National Heart, Lung, and Blood Institute Scientific Statement. Circulation, 2005; 112: 2735-2752.

12. Abbasi F, Brown BWB, Lamendola C, McLaughlin T, Reaven GM. Relationship between obesity, insulin resistance, and coronary heart disease. J Am Coll Cardiol, 2002; 40: 937-943.

13. McLaughlin T, Abbasi F, Lamendola C et al. Differentiation between obesity and insulin resistance in the association with C-reactive protein. Circulation, 2002; 106: 2908-2912.

14. Meigs JB, Wilson PWF, Fox CS et al. Body mass index, metabolic syndrome, and risk of type 2 diabetes or cardiovascular disease. J Clin Endocrinol Metab, 2006; 91: 2906-2912.

15. Olefsky JM, Farquhar JW, Reaven GM. Reappraisal of the role of insulin in hypertriglyceridemia. Am J Med, 1974; 57: 551-560.

16. Jeppesen J, Hollenbeck CB, Zhou M-Y et al. Relation between insulin resistance, hyperinsulinemia, postheparin plasma lipoprotein lipase activity, and postprandial lipemia. Arterioscler Thromb Vasc Biol, 1995; 15: 320-324.

17. Karpe F, Bard JM, Steiner G, Carlson LA, Fruchart JC, Hamsten A. HDLs and alimentary lipemia: studies in men with previous myocardial infarction at young age. Arterioscler Thromb, 1993; 13: $11-22$.

18. Swislocki ALM, Hoffman BB, Reaven GM, Insulin resistance, glucose intolerance and hyperinsulinemia in patients with hypertension. Am J Hypertens, 1989; 2: 419-423. 
19. Allemann Y, Horber FF, Colombo M et al. Insulin sensitivity and body fat distribution in normotensive offspring of hypertensive parents. Lancet, 1993; 341: 327-331.

20. TaittonenL,UhariM,NuutinenM,TurtinenJ,Pokka T, ÅkerblomHK. Insulin and blood pressure among healthy children. Am J Hypertens, 1996; 9:193-199.

21. ZavaroniI,MazzaS, Dall'AglioE, GaspariniP,PasseriM, Reaven GM. Prevalence of hyperinsulinaemia in patients with high blood pressure. J Intern Med, 1992; 231: 235-240.

22. Himsworth HP, Kerr RB. Insulin-sensitive and insulin-insensitive types of diabetes mellitus. Clin Sci, 1939; 4: 119-152.

23. Reaven GM. Insulin resistance in non-insulin-dependent diabetes mellitus. Does it exist and can it be measured? Am J Med, 1983; 74: $3-17$.

24. Lillioja S, Mott DM, Spraul M et al. Insulin resistance and insulin secretory dysfunction as precursors of non-insulindependent diabetes mellitus. N Engl J Med, 1993; 329: 1988-1992.

25. Tenenbaum A, Fisman EZ. "The metabolic syndrome... is dead": These reports are an exaggeration. Cardiovasc Diabetol, 2011; 10: 11.

26. Gami AS, Witt BJ, Howard DE et al. Metabolic syndrome and risk of incident cardiovascular events and death: a systematic review and meta-analysis of longitudinal studies. J Am Coll Cardiol, 2007; 49: 403-414.

27. Galassi A, Reynolds K, He J. Metabolic syndrome and risk of cardiovascular disease: A meta-analysis. Am J Med, 2006; 119: 812-819.

28. Mottillo S, Filion KB, Genest J et al. The metabolic syndrome and cardiovascular risk. A systematic review and meta-analysis. J Am Coll Cardiol, 2010; 56: 1113-1132.

29. Dohi T, Miyauchi K, Kasai T et al. Impact of metabolic syndrome on 10-year clinical outcomes among patients with acute coronary syndrome. Circ J, 2009; 73: 1454-1458.

30. Feinberg MS, Schwartz R, Tanne D et al. Impact of the metabolic syndrome on the clinical outcomes of non-clinically diagnosed diabetic patients with acute coronary syndrome. Am J Cardiol, 2007; 99: 667-672.

31. Herder M, Arntzen KA, Johnsen SH, Mathiesen EB. The metabolic syndrome and progression of carotid atherosclerosis over 13 years. The Tromso study. Cardiovasc Diabetol, 2012; 11: 77.

32. Leguisamo NM, Lehnen AM, Machado UF et al. GLUT4 content decreases along with insulin resistance and high levels of inflammatory markers in rats with metabolic syndrome. Cardiovasc Diabetol, 2012; 11: 100.

33. Hiuge A, Tenenbaum A, Maeda $\mathrm{N}$ et al. Effects of peroxisome proliferator-activated receptor ligands, bezafibrate and fenofibrate, on adiponectin level. Arterioscler Thromb Vasc Biol, 2007; 27: 635-641.

34. Ramli J, Calderon Artero P, Block RC, Mousa SA. Novel therapeutic targets for preserving a healthy endothelium: Strategies for reducing the risk of vascular and cardiovascular disease. Cardiol J, 2011; 18: 352-363.
35. Del Ben M, Baratta F, Polimeni L, Angelico F. Non-alcoholic fatty liver disease and cardiovascular disease: epidemiological, clinical and pathophysiological evidences. Intern Emerg Med, 2012; 7 (suppl. 3): 291-296.

36. Borges RL, Ribeiro AB, Zanella MT, Batista MC. Uric acid as a factor in the metabolic syndrome. Curr Hypertens Rep, 2010; 12: 113-119.

37. Garaulet M, Madrid JA. Chronobiology, genetics and metabolic syndrome. Curr Opin Lipidol, 2009; 20: 127-134.

38. Kong AP, Chan NN, Chan JC. The role of adipocytokines and neurohormonal dysregulation in metabolic syndrome. Curr Diabetes Rev, 2006; 2: 397-407.

39. Brady EM, Davies MJ, Hall AP, Talbot DC, Dick JL, Khunti K. An investigation into the relationship between sleep-disordered breathing, the metabolic syndrome, cardiovascular risk profiles, and inflammation between South Asians and Caucasians residing in the United Kingdom. Metab Syndr Relat Disord, 2012; 10: 152-158.

40. Tenenbaum A, Motro M, Fisman EZ et al. Bezafibrate for the secondary prevention of myocardial infarction in patients with metabolic syndrome. Arch Intern Med, 2005; 165: 1154-1160.

41. Erdem A, Uenishi M, Kukukdurmaz $Z$ et al. The effect of metabolic syndrome on heart rate turbulence in non-diabetic patients. Cardiol J, 2012; 19: 507-512.

42. Altuncu ME, Baspinar O, Keskin M. The use of short-term analysis of heart rate variability to assess autonomic function in obese children and its relationship with metabolic syndrome. Cardiol J, 2012; 19: 501-506.

43. Gurka MJ, Ice CL, Sun SS, Deboer MD. A confirmatory factor analysis of the metabolic syndrome in adolescents: An examination of sex and racial/ethnic differences. Cardiovasc Diabetol, 2012; 11: 128 .

44. Rasouli M, Nesarhosseini V Kiasari AM et al. The multiplicative interactions of leukocyte counts with some other risk factors enhance the prognostic value for coronary artery disease. Cardiol J, 2011; 8: 246-253.

45. Sullivan SD, Ratner RE. Should the metabolic syndrome patient with prediabetes be offered pharmacotherapy? Curr Diab Rep, 2011; 11: 91-98.

46. Rizzello M, De Angelis F, Campanile FC, Silecchia G. Effect of gastrointestinal surgical manipulation on metabolic syndrome: a focus on metabolic surgery. Gastroenterol Res Pract, 2012; 2012: 670418. doi: 10.1155/2012/670418. Epub 2012 Oct 22.

47. Navarese EP, Koziński M, Pafundi T et al. Practical and updated guidelines on performing meta-analyses of non-randomized studies in interventional cardiology. Cardiol J, 2011; 18: 3-7.

48. Grundy SM. Does the metabolic syndrome exist? Diabetes Care, 2006; 29: 1689-1692.

49. Yusuf $\mathrm{S}$, Hawken S, Ounpuu $\mathrm{S}$ et al. Obesity and the risk of myocardial infarction in 27,000 participants from 52 countries: A case-control study. Lancet, 2005; 366: 1640-1649. 